\title{
Applying Reactivation Tendency Analysis and Mohr-space to Evaluate Shear Strength Decrease and Anisotropy with Pre-existing Weakness(es) under Uniform Stress State
}

\author{
Hengmao Tong* \\ State Key Laboratory of Petroleum Resources and Prospecting, China University of Petroleum, Beijing 102249, China
}

\section{Article Info \\ *Corresponding author: Hengmao Tong \\ State Key Laboratory of Petroleum \\ Resources and Prospecting \\ China University of Petroleum \\ Beijing 102249, China \\ Tel: +86 1089734607 \\ Fax: +86 1089734158 \\ E-mail: tonghm@cup.edu.cn \\ tong-hm@163.com}

Received: February 22, 2017

Accepted: April 7, 2017

Published: April 13, 2017

Citation: Tong H. Applying Reactivation Tendency Analysis and Mohr-space to Evaluate Shear Strength Decrease and Anisotropy with Pre-existing Weakness(es) under Uniform Stress State. Int J Petrochem Res. 2017; 1(1): 31-39.

doi: 10.18689/ijpr-1000107

Copyright: @ 2017 The Author(s). This work is licensed under a Creative Commons Attribution 4.0 International License, which permits unrestricted use, distribution, and reproduction in any medium, provided the original work is properly cited.

Published by Madridge Publishers

\begin{abstract}
Understanding the mechanical controls on shear strength decrease due to preexisting weakness is a fundamental problem in tectonic studies. In this study, by applying reactivation tendency analysis theory, a theoretical framework and defined Shear-strength Coefficient $\left(f_{d}\right)$ are developed for evaluating the shear-strength decrease and anisotropies due to the presence of preexisting weakness(es). The proposed study managed to overcome the restrictions of previous studies assumption that a pre-existing weakness plane contains the intermediate stress $\left(\sigma_{2}\right)$ and vertical or horizontal orientations of principal stresses (Andersonian stress state). A new graphical technique (Mohr-space) was utilized to predict the shear-strength decrease and anisotropies caused by preexisting weakness(es). The Mohr-space technique made easier to visualize the state of stress and results of shear strength changes and able to build the quantitative and intuitive relationship between Shear-strength Coefficient $\left(f_{d}\right)$ and weakness relativeorientation $\left(\theta^{\prime}, \varphi^{\prime}\right)$, weakness mechanical properties $\left(C_{w}\right.$ and $\left.\mu_{w}\right)$ and relative $\sigma_{2}\left(\frac{\sigma_{1}-\sigma_{2}}{\sigma_{2}-\sigma_{3}}\right)$ in any uniform tri-axial stress state.. In this study, Shear-strength decrease and anisotropies of a rock sample are evaluated theoretically, and shear strength properties and deformation characteristics of a geological body with multiple pre-existing weaknesses are analyzed and predicted.
\end{abstract}

Keywords: Shear strength; preexisting weakness; reactivation tendency; Mohr-space; sandbox experiment.

\section{Introduction}

It is well documented that pre-existing weaknesses (fracture planes or faults, layering, fabrics etc.) can lead to decrease of shear strength and strength anisotropies [1-3], and the potential strength anisotropy created by pre-existing weakness is considerable [4]. Ranalli (1990) [5] proposed a unified quantitative model to evaluate strength anisotropies by the pre-existing weakness in terms of the three tectonic faulting regimes when the weakness plane contains the intermediate stress $\left(\sigma_{2}\right)$. However, to our knowledge strength anisotropies evaluation with pre-existing weakness is limited to two-dimensional cases (weakness plane containing $\sigma_{2}$ ) before slip-tendency theory was proposed [6]. However, strength anisotropies evaluation with slip-tendency [7] is confined to the qualitative analysis only. In addition, there is also some limitation in sliptendency theory, i.e. the principal stresses are oriented either vertically or horizontally, and the cohesive strength of all pre-existing weakness is neglected [6]. However, 
principal stress direction may depart significantly from vertical and horizontal with depth in the upper crust [8-9]. Furthermore, some weakness zones may possess cohesive strength, particularly in cemented faults, and properties of weaknesses may vary [4]. For a example, Sibson (1974) [10] showed that cementation of a fault zone can create 1.0-MPa cohesive strength or more. In this study, Shear-strength Coefficient $\left(f_{d}\right)$ is defined, and Reactivation Tendency analysis theory [11], and Mohr-space [12] are applied to evaluate strength decrease and anisotropies due to the presence of pre-existing weakness(es) with arbitrary azimuth in any uniform tri-axial stress state. Strength decrease and anisotropies caused by weakness may be intuitively simplified and quantitatively analyzed and evaluated with Mohr space.

\section{Mohr-space, pole $\left(\sigma_{n}, \tau_{n}\right)$ of any oriented plane in tri-axial stress state}

Mohr diagrams, which was introduced by Otto Mohr (1882), is one of the most used and useful tools in structural geology [13], and has been used extensively in mechanical problems, such as stress analysis, failure envelopes, fractures opening and reactivation [14] [15] [16] [17]. Although real three-dimensional Mohr diagrams do exist for any tri-axial stress state [13], Mohr-diagram is usually considered to be two-dimensional [13], which is well known as Mohr-circles. Mohr-cyclides, which can be used to represent any second rank tensor (including stress tensor) was introduced by Coelho and Passchier (2008) [13]. However, the stress components $\left(\sigma_{n^{\prime}} \tau_{n}\right)$ of any given plane are the most important, and the general diagrams of Mohr-cyclides are not so convenient to be used and prepared. In contrast, the Mohr space, which was proposed by Tong and Yin (2011) [11] can be used to express the normal stress and shear stress of a plane with an arbitrary azimuth in an arbitrary three-dimensional stress state.

In any given stress state, the pole $\left(\sigma_{n^{\prime}} \tau_{n}\right)$ of any plane (i.e. pre-existing weakness plane, defined by dip direction $\theta$ and dip angle $\varphi$ ) is either located on the three Mohr-circles (i.e. $P_{1}$ on large Mohr circle $\sigma_{1}-\sigma_{3^{\prime}} P_{2}$ and $P_{3}$ on small Mohr circles $\sigma_{1}$ $\sigma_{2}$ and $\sigma_{2}-\sigma_{3}$ respectively, Figure 1 ) or in the area (grey area in Figure 1) between large Mohr-circle and two small Mohrcircles(i.e. $P_{4}$ in Figure 2) in $\sigma_{n}-\tau_{n}$ coordinate system $[2,18]$. There is one to one correspondence relationship between any plane and its pole $\left(\sigma_{n^{\prime}} \tau_{n}\right)$ in $\sigma_{n}-\tau_{n}$ coordinate system. This space (i.e. the three Mohr-circles and the area between them) is called Mohr-space [11]. With the contour lines (pink and green dotted lines in Figure 2) of plane angles (pseudo-dip direction $\theta>$ and pseudo-dip angle $\varphi>\theta>=\alpha>+90^{\circ}$, where $\alpha>$ is the angle between the plane and the intersection line of the plane and $\sigma_{2}-\sigma_{3}$ plane; $\varphi>$ the angle between the plane and $\sigma_{2}-\sigma_{3}$ plane), the pole of the plane can be found in the Mohr-space. The relationship between $\theta \geqslant \varphi>\theta$, and $\varphi$ can be determined with transformation of coordinates [11], and the contour lines of angles can be compiled with equation (1) [11].

$$
\begin{aligned}
& \sigma_{\mathrm{n}}=\sigma_{1} \cos { }^{2} \varphi^{\prime}+\sigma_{2} \sin ^{2} \varphi^{\prime} \cos ^{2} \theta^{\prime}+\sigma_{3} \sin ^{2} \varphi^{\prime} \sin ^{2} \theta^{\prime} \\
& \left.\tau_{\mathrm{n}}=\left[\sigma_{1}^{2} \cos ^{2} \varphi^{\prime}+\sigma_{2}{ }^{2} \sin ^{2} \varphi^{\prime} \cos ^{2} \theta^{\prime}+\sigma_{3}{ }^{2} \sin ^{2} \varphi^{\prime} \sin ^{2} \theta^{\prime}\right)-\sigma_{\mathrm{n}}^{2}\right]^{1 / 2}
\end{aligned}
$$

Coordination definition and transformation can be seen in Tong and Yin (2011) [11].

Thus, by applying Mohr-space, normal stress $\left(\sigma_{n}\right)$ and shear stress $\left(\tau_{n}\right)$ of any plane can be conveniently and intuitively determined, and the changes of normal and shear stresses with plane relative-orientation $\left(\theta^{\prime}, \varphi\right)$ can be easily analyzed.

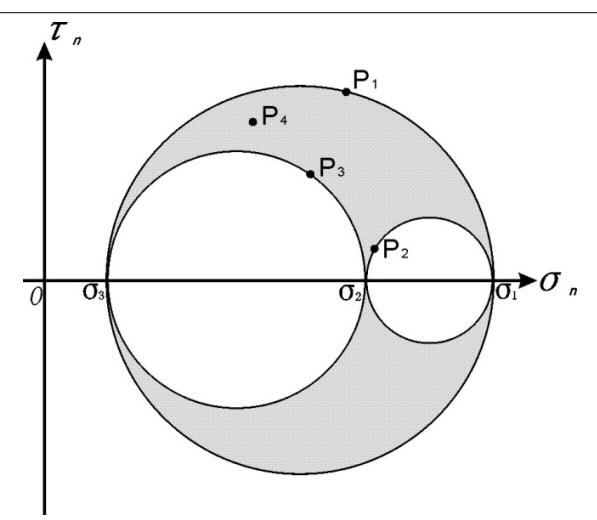

Figure 1. Diagram of three-axes stress Mohr circles. Planes parallel to $\sigma_{2}$ (i.e. $P_{1}$ ) constitute large Mohr circle $\sigma_{1}-\sigma_{3} ;$ planes parallel to $\sigma_{1}$ (i.e. $P_{2}$ ) or $\sigma_{3}$ (i.e. $P_{3}$ ) constitute small Mohr circle $\sigma_{1}-\sigma_{2}$ and $\sigma_{2}-\sigma_{3^{\prime}}$ respectively; the oblique planes (i.e. $P_{4}$ ) is locatedin the area (grey area) between large Mohr-circle and two small Mohr-circles.

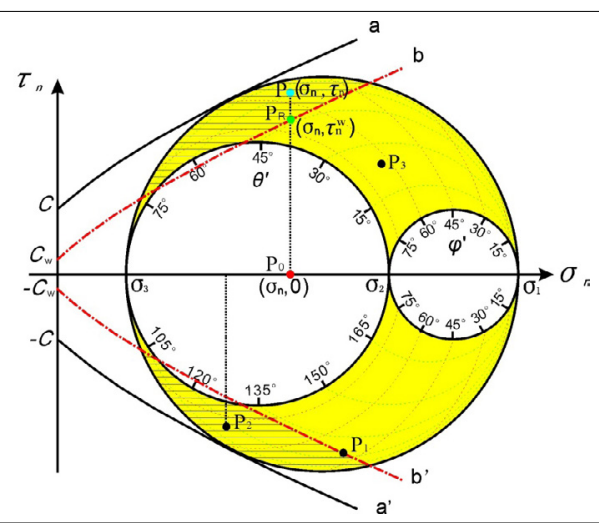

Figure 2. Diagram of Mohr space for the Reactivation Tendency Factor of a pre-existing weakness in critical stress state of CoulombMohr rupturing. Green dash line is contour of $\theta^{\prime}$, while pink dash line is contour of $\alpha^{\prime}$ (same is used in Figures 4, 5, 6, 8). A and a': Coulomb-Mohr rupture envelope lines, $b$ and $b^{\prime}$ : frictional strength lines of weakness. The striped yellow area is where weakness planes can be reactivated, while the other yellow area is where weakness can not be reactivated. Blue point $P\left(\sigma_{n^{\prime}} \tau_{n}\right)$ represents the pole of a weakness surface with given orientation, $\tau_{n}{ }^{W}$ is the critical shear stress of corresponding pre-existing weakness; Reactivation Tendency Factor $f_{a}=\left|\frac{\tau_{n}}{\tau_{n}^{w}}\right|=\frac{\mathbf{P}_{0}}{\mathbf{P}_{R}} \cdot \mathbf{P}_{1}$ is on the "weakness reactivation lines" and $f_{\mathrm{a}}\left(\mathrm{P}_{1}\right)=1.0, \mathrm{P}_{2}$ locates outside of the two "weakness reactivation lines" and $f_{a}\left(P_{2}\right)>1.0, P_{3}$ locates inside of the two "weakness reactivation lines" and $f_{a}\left(P_{3}\right)<1.0 . C, C_{w}$ are cohesive value of rock and weakness, respectively.

Reactivation Tendency Factor and its expressions in Mohrspace

Reactivation Tendency Factor $f_{a}=\left|\frac{\tau_{n}}{\tau_{n}^{w}}\right|=\left|\frac{\tau_{n}}{C_{w}+\mu_{w} \sigma_{n}}\right|$ (where $\tau_{n^{\prime}} \sigma_{n}$ are shear and normal stresses on the weakness plane, respectively; $\tau_{n}{ }^{w}$ is the corresponding rupture shear strength; $C_{w^{\prime}}$ and $\mu_{w}$ are cohesiveness and frictional coefficient 
of weakness plane, respectively), which is extended from Sliptendency [6] and is used to evaluate the reactivation likelihood of pre-existing weakness, is proposed by Tong and Yin[11] (2011). Reactivation Tendency Factor $\left(f_{a}\right)$ is determined by its relative-orientation $\left(\theta^{\prime}, \varphi^{\prime}\right)$, mechanical properties $\left(C_{w^{\prime}} \mu_{W}\right)$ of the weak plane, and the stress tensor. $f_{a}=1.0$ shows that the pre-existing weakness is in critical state of reactivation; when $f_{a}>1$, it has reactivated and when $f_{a}<1$, it is in a stable state. The weaknesses, which $f_{a} \geq 1.0$ in the critical stress state of Coulomb rupture, will reactivate one by one in progressive deformation according to their $\mathrm{f}_{\mathrm{a}}$ value order.

Applying Mohr-space, $f_{a}$ can be intuitively expressed accordingto the following steps. (1) The lines $\tau_{n}{ }^{w}= \pm\left(C_{w}+\mu_{w} \sigma_{n}\right)$ are two symmetrical straight lines (when $\mu_{w}$ is a constant)or curved lines ( $b$ and $b^{\prime}$ in Figure 2, when $\mu_{w}$ is variable) in $\sigma_{n}-\tau_{n}$ coordinate system, which were called "weakness reactivation lines" [19] or "shear strength line of weakness " [11], where $\left(0, C_{w}\right)$ is the starting point, $\mu_{w}$ is the slope of the line. If $C_{w}=0$ (weakness without cohesion), the line cross the point of origin. (2) The pole $\left(\sigma_{n^{\prime}} \tau_{n}\right)$ of a weakness plane ( $P$ in Figure 2 ) can be easily plotted in Mohrspace according to its relative-orientation $\left(\theta^{\prime}\right.$ and $\left.\varphi^{\prime}\right)$ and its $\sigma_{n^{\prime}} \tau_{n}{ }^{w}$ can be intuitively determined (Figure 2). As such, $f_{a}\left(\tau_{n} / \tau_{n}{ }^{w}\right)$ of the weakness plane can be easily and intuitively determined ( $P P_{0} / P_{R}$ in Figure 2). (3) When the pole of the weakness plane is on its "weakness reactivation line" $\left(P_{1}\right.$ in Figure 2$), f_{a}=1.0$ and the preexisting weakness is in the critical state of reactivation. However, when the pole is located outside the two "weakness reactivation lines" (striped yellow area in Figure 2, i.e. $P_{2}$ ), $f_{a}>1.0$ and the weakness plane has reactivated. When the pole is located inside the two weakness reactivation lines (yellow area without stripes in Figure 2 , i.e. $\left.P_{3}\right), f_{a}<1$ indicating that the plane is in a stable state.

\section{The definition of Shear-strength coefficient and its relationship to Reactivation Tendency Factor of pre-existing weakness}

As a weakness plane may be reactivated when stress is below Coulomb rupturing stress state, a pre-existing weakness will lead to decrease in shear strength [1] [2] [7]. However, the weakness with $f_{a}<1.0$ will not reactivate at critical stress of Coulomb rupture [11] according to Reactivation Tendency analysis theory. This means that not all weakness planes will lead to decrease in shear strength. - Therefore, in the following shear strength analysis, we only consider weaknesses with $f_{a} \geq 1.0$.

Consider a weakness plane $\left(\mathrm{P}_{\mathrm{w}}\right)$ with a normal $\mathrm{n}$ and its reactivation tendency factor $f_{a} \geq 1.0$ at critical uniform stress state $\left(\sigma_{1}, \sigma_{2}\right.$ and $\left.\sigma_{3}\right)$ of Coulomb rupture (Figure 3$) . P_{w}$ has been reactivated at critical stress state of Coulomb rupture [11], and its pole in Mohr-space is $\left(\sigma_{n^{\prime}} \tau_{n}\right)$ (Point A in Figure 3). With the same normal stress $\sigma_{n^{\prime}} P_{w}$ will be at critical state of reactivation when its shear stress is $\tau_{n}{ }^{w}$ (Point $B$ in Figure $3, \sigma^{\prime}{ }^{\prime}$ $\sigma_{2}^{\prime}$ and $\sigma_{3}^{\prime}$ are the three principal stresses) and $\tau_{n}{ }^{w}<\tau_{n}$. The large Mohr circle $\left(\sigma_{1}^{\prime}-\sigma_{3}^{\prime}\right.$ circle) of critical stress state of weakness reactivation is smaller than the $\sigma_{1}-\sigma_{3}$ Mohr circle of critical stress state of Coulomb rupture (Figure 3 ), that means $\sigma_{1}^{\prime}-\sigma_{3}^{\prime}<\sigma_{1}-\sigma_{3}$. Thus, in Mohr-space (Figure 3), it is very easy to understand that a weaknesswith $f_{a}>1$.0 will lead to decrease in shear strength.

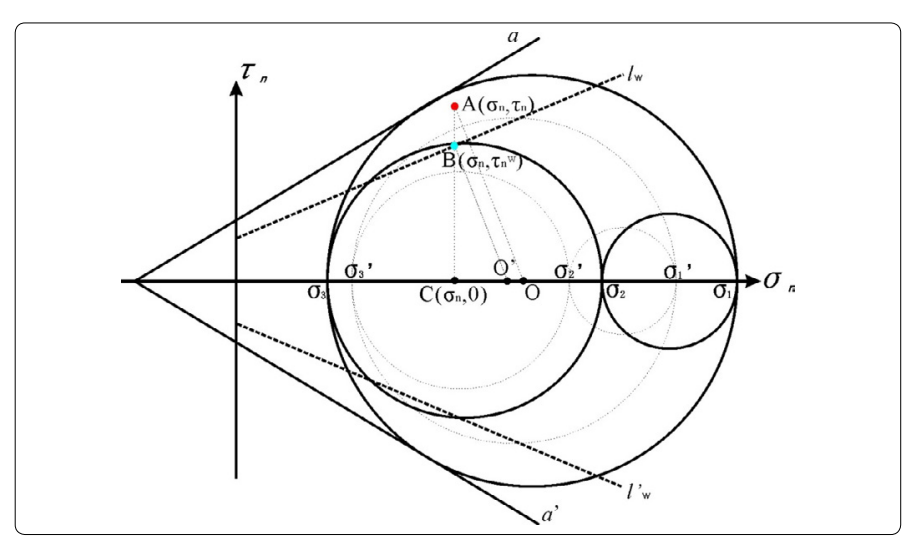

Figure 3. Diagram showing shear strength decrease due to pre-existing weakness. a and a': Coulomb-Mohr rupture envelope lines, $b$ and $b^{\prime}$ : frictional strength lines of weakness $(P) . \sigma_{1^{\prime}} \sigma_{2^{\prime}} \sigma_{3}$ are three principal stresses at critical stress state of Coulomb rupturing, and $\sigma_{1}^{\prime}{ }_{1}^{\prime} \sigma_{2^{\prime}}^{\prime} \sigma_{3}^{\prime}$ are three principal stresses at critical state of weakness reactivation. $A$ is the pole $\left(\sigma_{n^{\prime}} \tau_{n}\right)$ of a weakness $\left(P, f_{a}(P)\right.$ $>1.0)$ in $\left(\sigma_{1^{\prime}}, \sigma_{2^{\prime}}, \sigma_{3}\right)$ Mohr-space (solid line); $B$ is a point $\left(\sigma_{n^{\prime}}, w_{n}^{w}\right)$ on

the frictional strength line of the weakness (critical state of weakness reactivation) with the same normal stress $\sigma_{n^{\prime}}$ and also the pole of the weakness $(P)$ in $\left(\sigma^{\prime}{ }_{1}, \sigma_{2^{\prime}}^{\prime}, \sigma_{3}^{\prime}\right)$ Mohr-space (dashed line). $O$ and $O^{\prime}$ are center of large Mohr-circle $\sigma_{1}-\sigma_{3}$ and $\sigma_{1}^{\prime}-\sigma_{3^{\prime}}^{\prime}$

respectively. The shape of the two Mohr-space is the same ( $\left.\frac{\sigma_{1}-\sigma_{2}}{\sigma_{2}-\sigma_{3}}=\frac{\sigma_{1}^{\prime}-\sigma_{2}^{\prime}}{\sigma_{2}^{\prime}-\sigma_{3}^{\prime}}\right)$, the triangleS OAC and O'BC are similar. The Shear-strength coefficient $\left(f_{\mathrm{a}}\right)$ is defined as $f_{\mathrm{d}}=\left|\frac{\tau^{\mathrm{w}}{ }_{n}}{\tau_{n}}\right|=\frac{\mathrm{BC}}{\mathrm{AC}}$ and $f_{\mathrm{d}}=\frac{1}{f_{a}}$

Because shear strength is related to normal stress and increases with increasing of normal stress, relative instead of absolute shear strength is more useful to consider.In order to quantitatively evaluate the shear strength decrease due to the existence of a weakness, we define the parameter $f_{d}$ called Shear-strength coefficient $\left(f_{d}=\tau_{n}{ }^{w} / \tau_{n^{\prime}}\right.$ where $\tau_{n}{ }^{w}$ is the critical shear stress of weakness reactivation, $\tau_{n}$ is the shear stress on the same plane with the same normal stress $\sigma_{n}$ at critical stress state of Coulomb rupture $\left(\sigma_{1}, \sigma_{2}\right.$ and $\sigma_{3}$ stress state in Figure $3)$ ). It is easy to understand that the smaller Shear-strength coefficient $\left(f_{d}\right)$ is the larger decrease will be in shear strength.

When $f_{a} \geq 1.0, \tau_{n}{ }^{w}$ can never be greater than $\tau_{n}$ (as stress will drop down when weakness reactivates according to Reactivation Tendency analysis theory [20] [21]. Furthermore, when $f_{a} \leq 1.0$, which means that weakness will not be reactivated at critical stress state of Coulomb rupture and will not lead to shear strength decrease(i.e., $\left.f_{d}=1.0\right)$. Therefore, shear-strength coefficient can never be greater than $1.0\left(f_{d} \leq 1.0\right)$.

It is easy to find the following relationship between $f_{d}$ and Reactivation Tendency Factor $\left(f_{a}\right)$ at critical stress state of Coulomb rupturing-according to the definition of shearstrength coefficient and Reactivation Tendency analysis theory [11]

$\mathrm{f}_{\mathrm{d}}=\left|\frac{\tau_{\mathrm{n}}^{\mathrm{W}}}{\tau_{\mathrm{n}}}\right|=1 / \mathrm{f}_{\mathrm{a}}=\left|\frac{C_{w}+\mu_{w} \sigma_{n}}{\tau_{n}}\right| \mathrm{f}_{\mathrm{a}} \geq 1.0$
$\mathrm{f}_{\mathrm{d}}=1.0$
$\mathrm{f}_{\mathrm{a}}<1.0$

The relationship between Shear-strength Coefficient $\left(f_{d}\right)$ and the ratio of Differential stress can be derived at Coulomb rupture $\left(\left(\sigma_{1}-\sigma_{3}\right)_{c}=\sigma_{1}-\sigma_{3}\right.$ in Figure 3$)$ and at critical state of weakness reactivation $\left(\left(\sigma_{1}-\sigma_{3}\right)_{W}=\sigma_{1}{ }^{\prime}-\sigma_{3}{ }^{\prime}\right.$ in Figure 3$)$ according to the 
definition of Shear-strength Coefficient and using Mohr-space, and it means that $f_{d}=\left(\sigma_{1}-\sigma_{3}\right)_{W} /\left(\sigma_{1}-\sigma_{3}\right)_{C}$. As the relative position of the weakness pole in $\left(\sigma_{1}, \sigma_{2}, \sigma_{3}\right)$-Mohr-space (point A) and in $\left(\sigma_{1}{ }^{\prime}, \sigma_{2}{ }^{\prime}, \sigma_{3}{ }^{\prime}\right)$-Mohr-space (point $B$ ) is the same, $O A / O B$ is equal to the ratio of radius of the two big Mohr circle $\left(\frac{\sigma_{1}-\sigma_{3}}{2}\right.$ and $\left.\frac{\sigma_{1}{ }^{\prime}-\sigma_{3}{ }^{\prime}}{2}\right)$, which means that $O A / O B=\left(\sigma_{1}-\sigma_{3}\right) /\left(\sigma_{1}{ }^{\prime}-\sigma_{3}{ }^{\prime}\right)=\left(\sigma_{1}-\sigma_{3}\right) d\left(\sigma_{1}-\sigma_{3}\right)_{w}$. While two triangles $A O C$ and $B O^{\prime} C$ are similar, as a result, $B C / A C$ $=O B / O A$ and $f_{d}=\tau_{n}{ }^{w} / \tau_{n}=B C / A C=O B / O A=\left(\sigma_{1}-\sigma_{3}\right)_{C} /\left(\sigma_{1}-\sigma_{3}\right)$ ${ }{ }^{\prime}$ which means that $f_{d}=\left(\sigma_{1}-\sigma_{3}\right)_{d} /\left(\sigma_{1}-\sigma_{3}\right)_{W}$ when $f_{a} \geq 1.0$. So, $f_{d}(1 /$ $f_{a}$ ) is related to weakness-orientation $\left(\theta^{\prime}\right.$ and $\left.\varphi^{\prime}\right)$, mechanical properties $\left(C_{W^{\prime}} \mu_{W}\right)$ and the stress tensor according to Reactivation Tendency analysis theory [11], and it can be quantitatively calculated using equations 1 and 2-Since $f_{d}$ is related to weakness relative-orientation, weakness can lead to shear-strength anisotropies.

\section{Analysis of shear strength affection factors due to pre-existing weakness(es)}

Shear-strength coefficient $\left(f_{d}\right)$ is used to discuss how the related factors (mechanical properties and orientation of weakness, and $\frac{\sigma_{1}-\sigma_{2}}{\sigma_{2}-\sigma_{3}}$ ) affect shear strength due to preexisting weakness in triaxial stress state. As $f_{d}=1 / f_{a^{\prime}}$ and $f_{a}$ can be intuitively and conveniently expressed in Mohr-space, Mohrspace is applied to do these analysis.

\section{The relationship between $f_{d}$ and weakness relative- orientation $\left(\theta^{\prime}, \varphi^{\prime}\right)$}

It is easy to see that when $\theta^{\prime}=90^{\circ}$, and $\varphi^{\prime}= \pm \varphi_{0}^{\prime}= \pm$ $\left(45^{\circ}+0.5 \operatorname{arctg}\left(\mu_{w}\right)\left(\varphi_{0}^{\prime}\right.\right.$ is usually about $\left.60^{\circ}\right)$ (points $A$ and $A^{\prime}$ in Figure 4), $f_{a}$ reaches the highest value, whereas $f_{d}$ reaches the smallest value (Figure 4). Therefore, $\left(90^{\circ}, \pm \varphi_{0}^{\prime}\right)$ (two points) are the two weakest relative-orientation of the weakness plane(s). As $\theta^{\prime}$ decreases from $90^{\circ}$, and $\varphi^{\prime}$ deviates from $\varphi_{0^{\prime}}^{\prime} f_{d}$ will increase. In Mohr-space (Figure 4), the points of intersection between "weakness reactivation lines" and Mohr circles are demarcation points of weakness reactivation $\left(B_{1}, B_{2}\right.$ and $B^{\prime}{ }_{1}^{\prime} B^{\prime}{ }_{2}$ on $\sigma_{1}-\sigma_{3}$ large Mohr circle, $C_{1} C_{2}$ and $C^{\prime}{ }_{1} C^{\prime}{ }_{2}$ on $\sigma_{2}-\sigma_{3}$ small Mohr circle, Figure 4). The corresponding $\varphi^{\prime}$ of $B_{1^{\prime}} B_{2^{\prime}} B^{\prime}$ and $B_{2}^{\prime}$ is $\varphi_{1^{\prime}}^{\prime} \varphi_{2^{\prime}}^{\prime} \varphi_{1^{\prime}}^{\prime} \varphi_{2^{\prime}}^{\prime}$ and the corresponding $\theta^{\prime}$ of $C_{1^{\prime}} C_{2}$ and $C^{\prime}{ }_{1^{\prime}} C^{\prime}{ }_{2}$ is $\theta^{\prime}{ }_{1} \theta^{\prime}{ }_{2^{\prime}}\left(180^{\circ}-\theta^{\prime}{ }_{1}\right)$ and $\left(180^{\circ}-\theta_{2}^{\prime}\right)$ respectively. It is easy to find that when $\varphi^{\prime}<\varphi^{\prime}$, or $\varphi^{\prime}>\varphi^{\prime}{ }_{2^{\prime}}$ or $\theta^{\prime}<\theta^{\prime}$ or $\theta_{2}^{\prime}<\theta^{\prime}<180^{\circ}-\theta_{2}^{\prime}$ or $\theta^{\prime}>180^{\circ}-\theta^{\prime}{ }_{1}$, the weakness will all locate inside the two weakness reactivation lines (grey area in Figure 4) and $f_{a}$ will always $<1.0$, which means there exist critical angle $\varphi^{\prime}{ }_{1^{\prime}} \varphi_{2}^{\prime}$ for $\varphi^{\prime}$ and $\theta_{1^{\prime}} \theta^{\prime}{ }_{2}$ and $\left(180^{\circ}-\theta_{1}^{\prime}\right),\left(180^{\circ}-\theta_{2}^{\prime}\right)$ for $\theta^{\prime}$, when $\varphi^{\prime}<\varphi^{\prime}{ }_{1}$ or $\varphi^{\prime}>\varphi_{2^{\prime}}^{\prime}$ or $\theta^{\prime}<\theta_{1}^{\prime}$ or $\theta_{2}^{\prime}<\theta^{\prime}<180^{\circ}-\theta_{2}^{\prime}$ or $\theta^{\prime}>180^{\circ}-\theta^{\prime}{ }_{1}^{\prime} f_{a}$ will always $<1.0$ and the weakness cannot reactivate and will not lead shear strength decrease $\left(f_{d}=1.0\right)$.

Point $A$ and $A^{\prime}$ (Figure 4 ) are two Coulomb rupture points, and $f_{d}$ of the same oriented weakness plane is the lowest. Weakness plane with low $f_{d}$ value concentrates around point $A$ (or $A^{\prime}$ ) in the yellow area of Figure $4\left(\theta^{\prime}=90^{\circ} \sim 75^{\circ}\right.$ and $\left.\varphi^{\prime}=\varphi_{0}^{\prime} \pm 15^{\circ}\right)$. Change of $f_{d}$ shows that weakness relative-orientation is the predominant factor which leads to shear strength anisotropies. It is noteworthy tounderline that $\left(\theta^{\prime}, \varphi^{\prime}\right)$ of weakness is determined jointly by orientations of weakness and three axes of principal stresses.

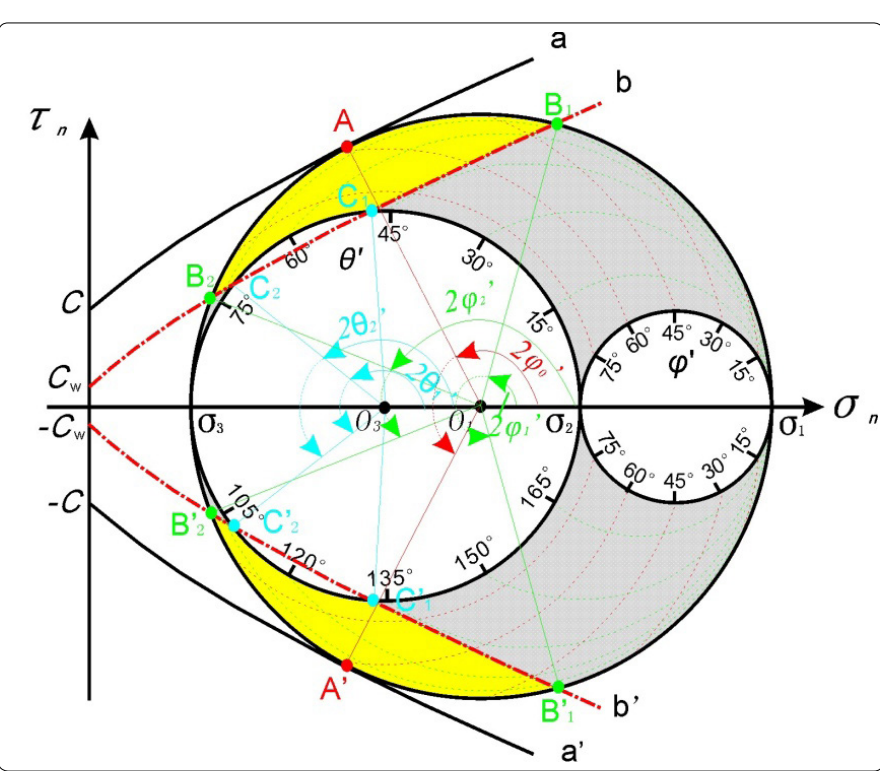

Figure 4. Diagrams of Mohr space showing the relationship between relative-orientation $\left(\theta^{\prime}, \alpha^{\prime}\right)$ and Shear-strength Coefficient $\left(f_{d}\right)$ of weakness. a and a': Coulomb-Mohr rupture envelope lines, $b$ and $b$ ': frictional strength lines of weakness. The horizontal lined yellow area is where weakness can be reactivated $\left(f_{d}<1.0\right)$ and the grey area is where weakness cannot be reactivated $\left(f_{d}=1.0\right)$. point $A$ and $A^{\prime}$ (both are $\left(90^{\circ}\right.$, $\left.\varphi_{0}{ }^{\prime}\right)$ ): Coulomb-Mohr rupture point, where the weakness is the weakest and with the lowest $f_{d}$. point $B_{1}, B_{2}$ and $B^{\prime}{ }_{1} B^{\prime}{ }_{2}$ are two pairs of points which weaknesses are at critical state of reactivation $\left(f_{d}=1.0\right)$ in $\sigma_{1}-\sigma_{3}$

Mohr circle, and $\varphi_{1}^{\prime}, \varphi_{2}^{\prime}$ and $180^{\circ}-\varphi^{\prime}{ }_{1}^{\prime} 180^{\circ}-\varphi_{2}^{\prime}$ are two pairs of corresponding critical $\varphi^{\prime}$ angle; point $C_{1^{\prime}} C_{2}$ and $C^{\prime}{ }^{2}{ }_{1} C^{\prime}$ are two pairs of points which weaknesses are at critical state of reactivation in $\sigma_{2}-\sigma_{3}$ Mohr circle, and $\theta_{1}^{\prime}, \theta^{\prime}$ are two of corresponding critical $\theta^{\prime}$ angle. $C^{2}, C_{w}$ is cohesive value of rock and weakness respectively.

The relationship between $f_{d}$ and weakness mechanical properties $\left(C_{w}\right.$ and $\left.\mu_{w}\right)$

In Mohr-space (Figure 5), it is easy to find that the change of mechanical property of a weakness plane $\left(C_{w}\right.$ and $\left.\mu_{w}\right)$ will cause the change of the position (determined by $C_{w}$ ) and the slope (determined by $\mu_{w}$ ) of weakness reactivation lines. It is easy to understand that $\mathbf{f}_{\mathbf{d}}$ will decrease with decreasing of $C_{w}$ and $\mu_{w}$
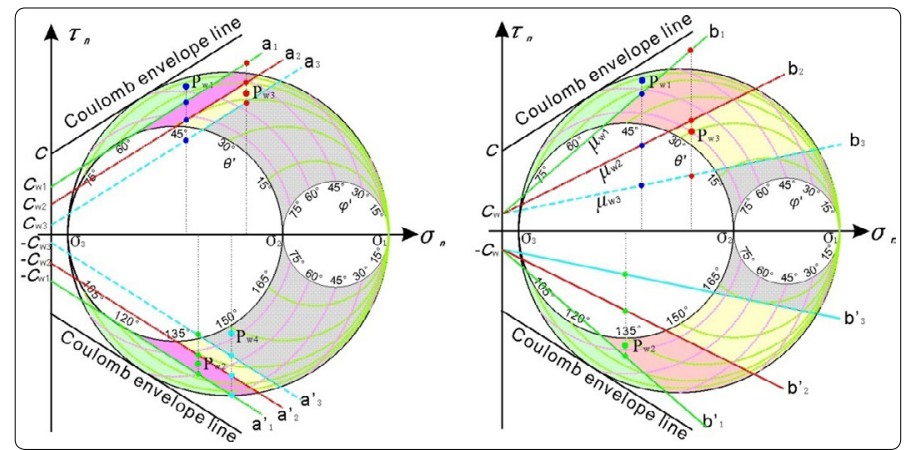

Figure 5. Diagrams of Mohr space to show how the mechanical property $\left(C_{w}\right.$ and $\left.\mu_{w}\right)$ of weakness affects its Shear-strength Coefficient $\left(f_{d}\right)$. a) The relationship between $C_{w}$ and $f_{d} \cdot a_{1}$ and $a_{1}{ }^{\prime}, a_{2}$ and $a^{\prime}, a_{3}$ and $a_{3}^{\prime}$ : three pairs of frictional strength lines of weakness with different cohesiveness and the same frictional coefficient of weakness. As $C_{w}$ decreases from $C_{w 1}$ to $C_{w 3}\left(C_{w 1}>C_{w 2}>C_{w 3}\right)$, the area where weakness can reactivate in Mohr space enlarges, and $f_{d}$ of the weaknesses with given orientation (such as weakness $P_{w 11^{\prime}} P_{w 2} P_{w 3}$ and $P_{w 4}$ ) decreases. b) The relationship between $\mu_{w}$ and $f_{d} \cdot b_{1}{ }^{w 1}$ and $b_{1}^{\prime \prime}, b_{2}$ and $b_{2}^{\prime}, b_{3}$ and $b_{3}^{\prime}$ : three pairs of frictional strength lines of weakness with different frictional coefficient and the same cohesiveness of weakness. As $\mu_{w}$ decreases from $\mu_{w 1}$ to $\mu_{w 3}\left(\mu_{w 1}>\mu_{w 2}>\mu_{w 3}\right)$, the area where weakness can reactivate in Mohr space enlarges, and $f_{d}$ of the weaknesses with given orientation decreases. 
Weakness mechanical properties are the predominant factors which lead to shear strength decrease. For example, low friction coefficient $\left(\mu_{w}<0.2\right.$ [22]) and no cohesion along the weakness may lead to more than $90 \%$ shear strength decrease $\left(\mathbf{f}_{\mathbf{d}}<0.1\right)$.

\section{The relationship between $f_{d}$ and relative value of $\sigma_{2}$}

Shear strength is also related to relative value of $\sigma_{2}$ $\left(\frac{\sigma_{1}-\sigma_{2}}{\sigma_{2}-\sigma_{3}}\right)$ [7]. However, our analysis in Mohr-space provided intuitive results which are easy to follow. It is easy to see that relative $\sigma_{2}\left(\frac{\sigma_{1}-\sigma_{2}}{\sigma_{2}-\sigma_{3}}\right)$ determines the shape of Mohr-space (Fig. 6). The relationship between $\frac{\sigma_{1}-\sigma_{2}}{\sigma_{2}-\sigma_{3}}$ and $\mathrm{f}_{\mathrm{d}}$ is complicated and depends on $\theta^{\prime}$ and $\varphi^{\prime}$, particularly $\varphi^{\prime}$ (Fig. 6). In general, $\varphi^{\prime}$ can be divided into 3 intervals: $\varphi^{\prime} \geq 70^{\circ}, 40^{\circ}<\varphi^{\prime}<70^{\circ}$, and $\varphi^{\prime} \leq 40^{\circ}$. When $\varphi^{\prime} \geq 70^{\circ} f_{d}$ will increase $\left(P_{w 1}\right.$ in Figure 6). When $40^{\circ}<\varphi^{\prime}<70^{\circ}$, $f_{d}$ decreases a little $\left(P_{w 3^{\prime}}, P_{w 4}\right.$ and $P_{w 5}$ in Figure 6), and when $\varphi^{\prime} \leq 40^{\circ}, f_{d}$ changes little $\left(P_{w 6}\right.$ and $P_{w 7}$ in Figure 6). These three cases are valid when $\sigma_{2}$ decrease(or $\frac{\sigma_{1}-\sigma_{2}}{\sigma_{2}-\sigma_{3}}$ increases, Fig. 6). As such, the change of $\sigma_{2}$ should not be ignored in shear strength analysis in the presence of pre-existing weakness. However, when $\varphi^{\prime} \geq 70^{\circ}$ or $\varphi^{\prime} \leq 40^{\circ}$ (particularly when $\varphi^{\prime} \leq 40^{\circ}$ ), most of the weaknesses usually cannot be reactivated at critical stress state of Coulomb rupturing and thus doesnot lead shear-stress decrease $\left(f_{d}=1.0\right)$. As a result, the effect of relative $\sigma_{2}$ is most prominant only in the interval $40^{\circ}<\varphi^{\prime}<70^{\circ}$; under normal circumstances, $f_{d}$ will decrease a little with decreasing $\sigma_{2}$ (Fig. 6).

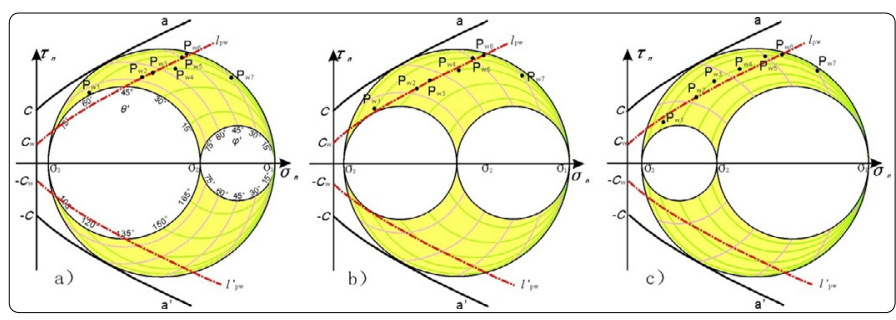

Figure 6. Diagrams of Mohr space to show the relationship between relative $\sigma_{2}$ and $\mathrm{f}_{\mathrm{d}}$ of weakness. a) $\frac{\sigma_{1}-\sigma_{2}}{\sigma_{2}-\sigma_{3}}=05$; b) $\frac{\sigma_{1}-\sigma_{2}}{\sigma_{2}-\sigma_{3}}=1.0$; c) $\frac{\sigma_{1}-\sigma_{2}}{\sigma_{2}-\sigma_{3}}=2.0$. a and a': Coulomb-Mohr rupture envelope lines, $\mathrm{I}_{\mathrm{pw}}$ and $\mathrm{I}_{\mathrm{pw}}^{\prime}$ : frictional strength lines of weakness. As $\sigma_{2}$ decreases ( $\frac{\sigma_{1}-\sigma_{2}}{\sigma_{2}-\sigma_{3}}$ increases, from Figure a to $\mathrm{c}$ ), the shape of Mohr space will change. The relationship between $\sigma_{2}$ and $f_{d}$ is a little complicated and is related to $\varphi^{\prime}$. There are three intervals: $\varphi^{\prime} \geq 70^{\circ}, 40^{\circ}<\varphi^{\prime}<70^{\circ}$ and $\varphi^{\prime} \leq 40^{\circ}$. When $\varphi^{\prime} \geq 70^{\circ}, 40^{\circ}<\varphi^{\prime}<70^{\circ}$ and $\varphi^{\prime} \leq 40^{\circ}$, $f_{d}$ will increase

$\left(P_{w 1}\right)$, decrease $\left(P_{w 3^{\prime}} P_{w 4}\right.$ and $\left.P_{w 5}\right)$, and change little $\left(P_{w 6}\right.$ and $\left.P_{w 7}\right)$ respectively with decreasing of $\sigma_{2}$ (or increasing of $\left.\frac{\sigma_{1}-\sigma_{2}}{\sigma_{2}-\sigma_{3}}\right)$. The $\left(\theta^{\prime}\right.$,

$\left.\varphi^{\prime}\right)$ of weakness $P_{w 1^{\prime}} P_{w 2^{\prime}} P_{w 3^{\prime}} P_{w 4^{\prime}} P_{w 5^{\prime}} P_{w 6}$ and $P_{w 7}$ is $\left(30^{\circ}, 82^{\circ}\right),\left(45^{\circ}\right.$, $\left.88^{\circ}\right),\left(45^{\circ}, 60^{\circ}\right),\left(60^{\circ}, 60^{\circ}\right),\left(45^{\circ}, 45^{\circ}\right),\left(15^{\circ}, 38^{\circ}\right)$ and $\left(45^{\circ}, 30^{\circ}\right)$ respectively, and their mechanical property are the same.

Mechanical properties and relative-orientation, which are the governing factors of shear strength decrease and strength anisotropies, respectively, are internal factors, while $\frac{\sigma_{1}-\sigma_{2}}{\sigma_{2}-\sigma_{3}}$ is an external factor and not so important according to the above analysis,. When the relative-orientation $\left(\theta^{\prime}, \varphi^{\prime}\right)$ and mechanical properties $\left(C_{\mathrm{w}^{\prime}}, \mu_{W}\right)$ of pre-existing weakness, and $\frac{\sigma_{1}-\sigma_{2}}{\sigma_{2}-\sigma_{3}}$ are given, the Shear-strength Coefficient can be quantitatively evaluated in Mohr-space.

\section{Shear strength evaluation for rock samples and geological bodies: theoretical analysis and verification}

There are many kinds of pre-existing weakness, such as faults, geologic contacts, bedding and foliation that affect shear strength [23] [24] [25]. Based on their structure, Morley (2002) [26] divided weaknesses into two types: "discrete" and "pervasive". Based on the value of cohesive strength, Tong and Yin (2011) [11] divide weaknesses into "strong weakness" (with relatively large cohesive strength, such as bedding, foliation etc.) and "weak weakness" (with relatively small or zero cohesive strength (i.e. ignorable), such as faults, fracture planes etc.). "Discrete" weakness is usually a "weak weakness", and "pervasive" weakness probably is a "strong weakness". We will evaluate the effect of "strong weakness" and "weak weakness" on shear strength in rock samples, and analyze deformation sequences with multiple pre-existing weaknesses in geological bodies.

\section{Rock samples}

"Pervasive" weaknesses (or "strong weakness", bedding in sedimentary rocks, foliation in metamorphic rocks) do exist in rocks. There may also exist"pervasive" weaknesses in magmatic rocks with ductile deformation or flow foliation. In homogeneouslooking rock samples there may exist "pervasive" (particularly in sedimentary or metamorphic rocks) and /or "discrete" weaknesses ("weak weakness", internal small or micro-fracture) leading to shear strength anisotropies.

In order to quantitatively evaluate shear strength decrease and anisotropies of rock samples with "pervasive" or "discrete" weakness, the situations of $11 C_{w}=0.5 C, 0.33 C$ and $0.2 C$ for "pervasive"weakness, and $C_{\mathrm{w}}=0$ for "discrete" weakness, and (2) $\frac{\sigma_{1}-\sigma_{2}}{\sigma_{2}-\sigma_{3}}=0.5,1.0$ and 2.0 are considered (let $\mu_{W}=\mu=0.6$, $C=20 \mathrm{MPa})$. Applying Mohr-space, the calculated values of Shear-strength Coefficient can be seen in Table 1. For other $C$ values, the result is completely the same, while the result will change a little bit as $\mu\left(\mu_{W}\right)$ changes (if $\left.\mu_{W}=\mu\right)$. However, if $\mu_{W}$ changes while $\mu$ isconstant, $\mathrm{f}_{\mathrm{d}}$ will change proportionally with $\mu_{W}$. The results show thata "discrete" weakness may lead to more than $80 \%$ maximum drop of shear strength, while "pervasive" weakness usually leads to $20-60 \%$ maximum shear strength decrease for rock samples.

Haimson and Rudnicki (2010) [27] conducted true tri-axial compression tests on siltstone samples, and showed that shear strength is related to $\sigma_{2}$. Although the orientation of siltstone bedding is not mentioned in the paper, we speculate that the shear strength change is caused by "pervasive" weakness (siltstone bedding). If bedding does not lead to mechanical anisotropy (i.e. when it is mechanically homogeneous), shear strength will not change with $\sigma_{2}$. The tri-axial compression tests of Haimson and Rudnicki (2010) [28] may be the verification for the above theoretical analysis of dependece of shearstrength on 
$\sigma_{2}$. Naturally, availability of more experiment data is needed.

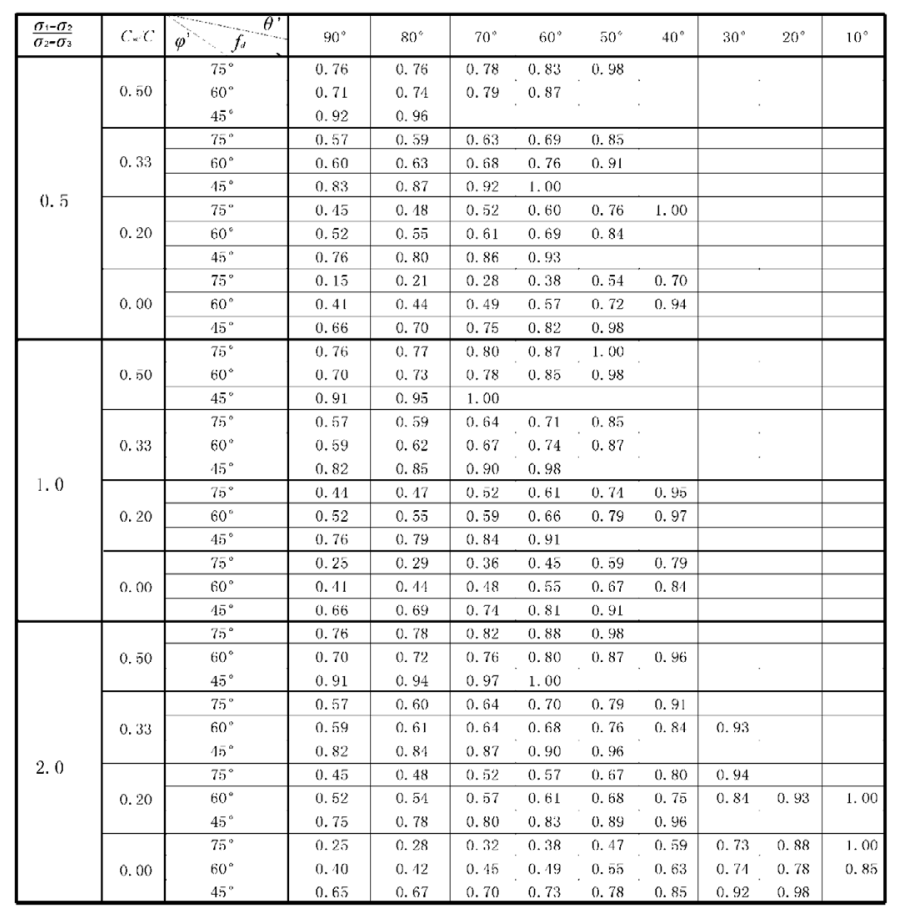

Table 1: Shear-strength Coefficient $\left(f_{\mathrm{d}}\right)$ value in different value of $C_{w^{\prime}}$ relative $\sigma_{2^{\prime}} \theta^{\prime}$ and $\varphi^{\prime}$.

In the presence of multiple pre-existing weaknesses in a rock sample, each weakness has its own Shear-strength Coefficient. The overall Shear-strength Coefficient is determined by and is equal to that of the weakest weakness, where the Shear-strength Coefficient is the smallest, if interactions of weaknesses are ignored.

\section{Geological bodies and deformation sequence}

There are the two main differences between geological bodies and rock samples when the size and locationof weakness are concerned. It is possible to prepare relatively homogeneous small rock samples (it is necessary for regular rock mechanics test). However, it is inevitable that there are pre-existing weaknesses more or less in the geological bodies, which will lead to shear strength decrease (shear strength is much smaller than that of rock samples [1] [2] [7].

On a larger scale, there may or probably exist multiple weaknesses [13] in geological bodies (i.e. rift basins, orogenic belts, or a part of them). Different relative-orientation of the weaknesses and/or their mechanical properties will lead to different $f_{d}$ of weakness. The relative-orientation and/or mechanical properties may also vary greatly along large scale pre-existing weakness (i.e. big pre-existing faults) and will lead to different $f_{d}$ in different segments along the same weakness.

Unlike rock samples, in the presence of multiple pre-existing weaknesses in geological bodies, one of the weaknesses reactivation will lead to stress drop and form local stress field only in the area along and near the weakness [20] [21] instead of the whole geological body. Differential stress within other areas of the body away from the weakness can increase with progressive deformation until another weakness reactivates or Coulomb rupturing occurs. In otherwords, a weakness can lead to shear strength decrease only within a part of geological bodies (along and near a "prefered" weakness). As a result, in the condition of homogeneous regional stress field, it is easy to understand that the weaknesses, which are away from each other and $\mathrm{f}_{d}<1.0$, can reactivate according to their $f_{d}$ values (from small to large) in the progressive deformation: the weakest weakness with the smallest $f_{d}$ value reactivates first, then the second weakest, and so on. Coulomb rupturing will occur at last in the area away from the weaknesses.

In order to verify the above statements, a sandbox model is run, where multiple pre-existing weaknesses are built away from each other. The $f_{d}$ values of these weaknesses can be quantitatively determined, and the regional stress field can be regarded as homogeneous.

In the models, three pre-existing weakness planes $\left(\mathrm{P}_{\mathrm{w} 1^{\prime}} \mathrm{P}_{w 2}\right.$ and $\left.P_{w 3}\right)$, which are oblique to the extension direction $\left(\sigma_{3}\right)$, with $\left(\varphi^{\prime}, \theta^{\prime}\right)=\left(80^{\circ}, 55^{\circ}\right),\left(60^{\circ}, 70^{\circ}\right)$ and $\left(30^{\circ}, 49^{\circ}\right)$ respectively, are set. The weakness planes are represented by a $80 \mathrm{~mm}$ wide slice of thin sheets of paper inserted into the base of a homogeneous layer ( $8.0 \mathrm{~cm}$ thick) of dry quartz sand (Fig. 7). Using these sheets of paper allowed localizing fault initiation, and it was easy to determine friction angle between the paper and loose sand. The friction angle of dry quartz sand is $31^{\circ}$, while the friction angle of sand with paper is $20^{\circ}$. So the $\mu$ and $\mu_{\mathrm{w}}$ is $0.60\left(=\operatorname{tg} 31^{\circ}\right)$ and 0.36 $\left(=\operatorname{tg} 20^{\circ}\right)$. The cohesion of dry sand is very small, so $C$ and $C_{w}$ are both assumed to be equal to 0 . As a result, the relative Reactivation Tendency Factor of the three weakness planes is $\mathrm{f}_{\mathrm{a}}$ $=1.59>f_{a 2}=1.42>1.0>f_{a 3}=0.85$ in the critical stress state of Coulomb rupture (Fig. 8), and Shear-strength Coefficient $f_{d 1}$ $=0.63<f_{d 2}=0.70<1.0=f_{a 3}$.

With the above model (Figure 7), the weakness, with Shearstrength Coefficient $f_{d 1}<1.0$, reactivated to form several faults, one after another, in the progressive extension as predicted, and Coulomb ruptures faults form at last (Figure 9). The deformation in the progressive extension is summarized in the following:
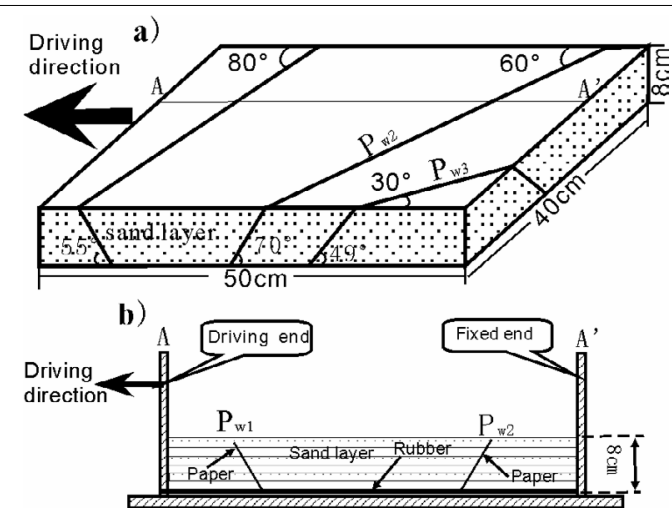

Figure 7. Experiment design of sandbox modeling. (a) Skeleton figure of experimental model. It is $40 \mathrm{~cm}$ wide, $50 \mathrm{~cm}$ long and $8 \mathrm{~cm}$ thick. $P_{w 11^{\prime}} P_{w 2^{\prime}}$ and $P_{w 3}$ are weakness planes which is oriented oblique to the extension direction, with $\left(\theta^{\prime}, \varphi^{\prime}\right)$ are $\left(80^{\circ}, 55^{\circ}\right),\left(60^{\circ}, 70^{\circ}\right)$ and $\left(30^{\circ}, 49^{\circ}\right)$ respectively, and represented by paper being inserted into the homogeneous dry sand. Elastic rubber is under the sand layer and connected to driving end. (b) Section of $A A^{\prime}$ in figure (a).

The weakness plane with the smallest Shear-strength Coefficient $\left(P_{w 1}\right)$ reactivated to form a fault at first (fault number 1, Fig. $9 b-1)$. Then, the second weakness plane $\left(P_{w 2}\right)$ reactivated to form a fault (fault number 2 , Fig. $9 b-1$ ). In the 
middle stage (after the weakness reactivated and before the fault near perpendicular to extension direction formed), weakness related faults which are parallel (or sub-parallel) to and controlled by $\mathrm{P}_{\mathrm{w} 1}$ or $\mathrm{P}_{\mathrm{w} 2}$ (antithetic and synthetic faults, fault numbers 3-8in Figures $22 b-2$ and $b-3$ ) began to develop near the weakness plane area. In the final stage (Fig. 9b-3), faults near perpendicular to the extension direction (small faults in Figure 9b-3) began to develop. While the third weakness plane, which Shear-strength Coefficient $f_{d 3}=1.0\left(f_{a 3}\right.$ $=0.85<1.0$, Fig. 8), does not reactivate as predicted. Similar experiments carried out by Tong and Yin (2011) [11] and Tong et al. (2014) [28] showed the same results.

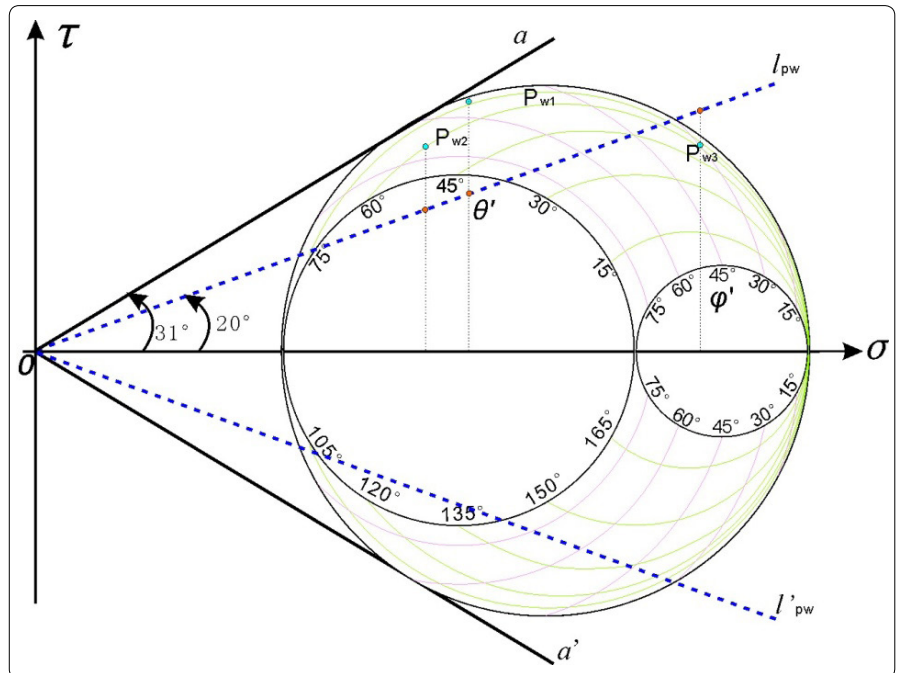

Figure 8. Diagram of Mohr space at critical stress state of Coulomb rupture of dry sand and the Shear-strength coefficient of the three weakness planes in Figure 7. a: Coulomb-Mohr rupture envelope line, $I_{\mathrm{pw}}$ : friction strength line of the weakness; the content of Mohr space is the same within Figure 3.Point $P_{w 1}\left(80^{\circ}, 55^{\circ}\right), P_{w 2}\left(60^{\circ}, 70^{\circ}\right)$ and $P_{w 3}\left(30^{\circ}, 49^{\circ}\right)$ represent the position of the three weakness planes respectively, and the corresponding point (small point with the same color) on the friction strength line of the weakness represent their own frictional strength. Point " $A$ " is the point of Coulomb-Mohr rupture.

In general, a phase of deformation indicated by the initiation of a new fault trend is attributed to a specific stress regime. However, our analysis suggests that in a region where the magnitude of differential stresses progressively increase while their directions are kept constant, multiple phases of fault initiation with different trends can occur. This could be due to reactivation of pre-existing weaknesses. The sequence of reactivation of any preexisting weakness is predictable according to the relative-orientation and mechanical properties of the weak planes.

It is not easy to estimate the shear strength of pre-existing weaknesses in the field. Even though, the relative-orientation of a weakness may be determined accurately in some circumstances (when high quality 3D seismic data is available, and fault systems in shallow level can be mapped accurately), mechanical properties of the weakness $\left(C_{w^{\prime}} \mu_{W}\right)$ are not easily determined. However, if the sequence of faulting in the geologic record is known, this information may be reversely used for assessing the mechanical properties of the preexisting weaknesses at the time of their reactivation.

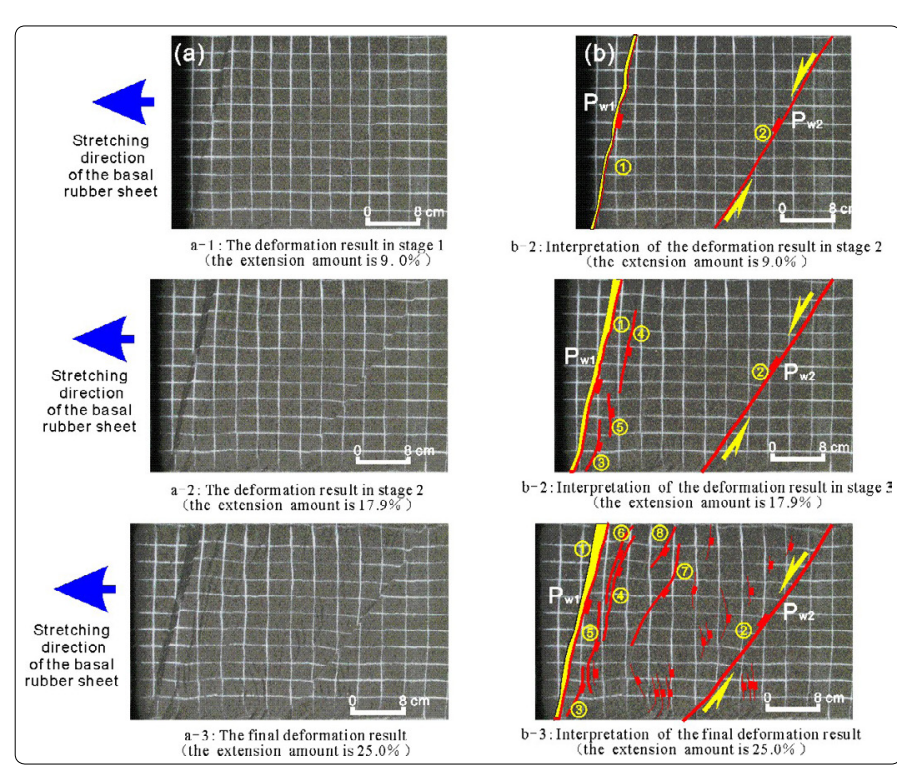

Figure 9. Sandbox modeling results and interpretation. (a) Experiment results. (b) Interpretation of experiment result. The number in the figure is the fault formation order. $a-1$ and $b-1: P_{w 1}$ and $P_{w 2}$ reactivated to form a fault (Fault (1) and (2)), there is no other fault yet. a-2 and b-2: Synthetic faults (Fault (3, (4) and (5))of

$P_{w 1}$ reactivated fault formed, the displacement of $P_{w 1}$ and $P_{w 2}$ reactivated fault increased. a-3: More synthetic faults (Fault (6), (7) and (8)) of $P_{w 1}$ reactivated fault formed, and the faults near perpendicular to extension direction formed at last, the displacement of $\mathrm{P}_{\mathrm{w} 1}$ and $\mathrm{P}_{\mathrm{w} 2}$ reactivated fault increased continuously and their length kept unchanged, while $\mathrm{P}_{\mathrm{w} 3}$ kept inactive.

\section{Discussion}

Although this study is expanding the work of Ranalli (1990) [5] and Morris et al [7], our newly defined Shearstrength Coefficient, newly developed graphical techniqueMohr-space and the underlying theoretically framework, which is based on Reactivation Tendency analysis theory (Tong and Yin, 2011), provide a much general and intuitive treatment of the shear strength decrease and anisotropies caused by pre-existing weakness(es). Specifically, the assumption that the weakness plane containing the intermediate stress $\left(\sigma_{2}\right)$ in Ranalli's (1990) [5] analysis can now be neglected. Finally, we predicted that weaknesses will reactivate sequentially according to the Shear- strength coefficient values order (from small to large) and new fractures (Coulomb rupture) form at last in the progressive deformation. It was verified by a simple sandbox experiment.

Both in this study and that of Ranalli (1990) [5], it is assumed that the preexisting weakness must be planar. This assumption, however, does not prevent the application of the analysis developed in this study to non-planar weaknesses, as curved surfaces can always be divided into approximately small planar segments. Another important assumption in our study is that fault formation, propagation and activity do not affect the regional stress distribution. This assumption is clearly an oversimplification, as both detailed analysis of faultzone evolution and regional modeling show that frictional sliding on faults is capable of creating local stress fields near the faults that are different from the regional stress field [29] [30]. However, the results of our sandbox experiment imply 
that the effect of activating preexisting weakness does affect local stress, but is minimal in changing regional stress. More research is clearly needed to address this problem.

Possible complex interactions between faults that lie near one another were not considered in our model. As stress concentrates at crack tips, it is expected that complex stress fields can be induced by the presence of cracks or weaknesses under uniform regional stress [30] (e.g., Gudmundsson et al., 2009). Thus, our model should be considered as an idealized conceptual guide, which can only be used in realistic situations when the above factors are considered.

Despite the complexities discussed above, our analysis does provide a new insight into the temporal evolution of multiple pre-existing weaknesses with different Shearstrength coefficient. Our analysis suggests that in a region with a progressive increase in the magnitude of differential stresses while the directions of the principal stresses are not changed, multiple phases of fault initiation with different trends can be generated. On the other hand, the results of theoretical analysis will provide some information and clues to understand actual shear strength decrease and anisotropies due to the pre-existing weaknesses.

\section{Conclusion}

Shear-strength coefficient $\left(f_{d}\right)$, which is defined to evaluate the shear strength decrease due to the presence of preexistingweaknesses, is determined by the orientation and mechanical properties of weakness(the intrinsic factors) and stress tensor (the external factors), and can be calculated quantitatively. It also can be expressed intuitively/graphically in Mohr-space. The results of theoretical analysis in Mohrspace show that:

(1)Weakness relative-orientation $\left.\left(\theta^{\prime}, \varphi\right)^{\prime}\right)$, which is determined jointly by orientation of the weakness and three principal stress axis, is the predominant factor leading to shear strength anisotropies. $\left(0^{\circ}, \varphi_{0}^{\prime}\right)$ and $\left(180^{\circ}, \varphi_{0}^{\prime}\right)$ are two relativeorientations with the lowest $f_{d^{\prime}}$. As $\theta^{\prime}$ increases from $0^{\circ}$ or decreases from $180^{\circ}$, and $\varphi^{\prime}$ deviates from $\varphi_{0^{\prime}}{ }^{\prime} f_{d}$ will increase. There are critical angles $\varphi^{\prime}{ }_{1}, \varphi_{2}^{\prime}$ and $\theta^{\prime}{ }_{1}, 180^{\circ}-\theta^{\prime}{ }_{1}$, when $\varphi^{\prime}{ }_{1}<\varphi^{\prime}<$ $\varphi_{2}^{\prime}$ or $\theta_{1}^{\prime}<\theta^{\prime}<180^{\circ}-\theta^{\prime}{ }_{1} f_{d}=1.0$. Low $f_{d}$ value of weakness plane concentrates in the area around $\theta^{\prime}=0^{\circ} \sim 15^{\circ}$ or $165^{\circ} \sim 180^{\circ}$ and $\varphi^{\prime}=\varphi_{0}^{\prime} \pm 15^{\circ}$.

(2)Weakness mechanical properties $\left(C_{w}\right.$ and $\left.\mu_{w}\right)$ are the predominant factorsthat lead to shear strength decrease. "Discrete" weaknesses may suffer more than $80 \%$ decrease in maximum shear strength, while "pervasive" weaknesses usually suffer 20-60\% decrease in maximum shear strength.

(3)The effect of relative $\sigma_{2}\left(\frac{\sigma_{1}-\sigma_{2}}{\sigma_{2}-\sigma_{3}}\right)$ to shear strength is a little complicated, and is related to $\theta^{\prime}$ and $\varphi^{\prime}$, particularly $\varphi^{\prime}$. The effect of relative $\sigma_{2}$ is most prominant only in the interval $40^{\circ}<\varphi^{\prime}<70^{\circ}$. Under normal circumstances, $f_{d}$ will decrease a little with increasing of $\frac{\sigma_{1}-\sigma_{2}}{\sigma_{2}-\sigma_{3}}$.

In a region with a progressive increase in the magnitude of differential stresses acting on multiple pre-existing weaknesses, while the directions of the principal stresses maintain the same, multiple phases of fault initiation with different trends can be generated with predicted sequence according to their shear-strength coefficient (from small to large). This theoretical prediction wasverified by the results of a sandbox model.

\section{Acknowledgement}

We would like to thank graduate students Mingyang WANG and Hua wu HAO for drawing the figures of the Mohr space and their help to complete the sandbox model.This study was supported by China National Major Project of Oil and Gas (2016ZX05024-005-004 \& 2011ZX05023-0040122011zx05006-006-02-01) and China Natural Science Foundation (Grant No. 41272160\&40772086). HAK is supported by the Swedish Research Council.

\section{References}

1. Jaeger JC. Shear fracture of anisotropic rocks. Geol. Mag. 1960; 97: 65-72.

2. Jaeger J C, Cook NGW. Fundamentals of rock mechanics (third edition): London, United Kingdom, Chapman and Hall, 1979; 593.

3. Ranalli G. Rheology of the Earth: Deformation and Flow Processes in Geophysics and Geodynamics. Allen \& Unwin, London. 1987.

4. Morley C K, Haranya C, Phoosongsee WS. J Structural Geology. 2004; 26(10): 1803-29. doi: 10.1016/j.jsg.2004.02.014

5. Ranalli G, Yin ZM. Critical stress difference and orientation of faults in rocks with strength anisotropies: the two-dimensional case. J Structural Geology. 1990; 12(8): 1067-71. doi: 10.1016/0191-8141(90)90102-5

6. Morris AP, David AF, Brent $H$. Slip-tendency analysis and fault reactivation. Geology. 1996; 24(3): 275-78. doi: 10.1130/0091-7613

7. Morris AP, Ferrill DA. The importance of the effective intermediate principal stress $\left(\sigma_{2}\right)$ to fault slip patterns. J Structural Geology. 2009; 31(9): 950-59. doi: 10.1016/j.jsg.2008.03.013

8. Spencer JE, Chase CG. Role of crustal flexure in initiation of low-angle normal faults and implications for structural evolution of the Basin and Range province. J. Geophys. Res. 1989; 94: 1765-75. doi: 10.1029/ JB094iB02p01765

9. Yin A. Origin of regional, rooted low-angle normal faults: a mechanical model and its tectonic implications. Tectonics.1989; 8(3): 469-82. doi: 10.1029/TC008i003p00469

10. Sibson RH. Frictional constraints on thrust, wrench, and normal faults. Nature. 1974; 249: 542-44. doi: 10.1038/249542a0

11. Tong $H$, Yin A. Reactivation tendency analysis: A theory for predicting the temporal evolution of preexisting weakness under uniform stress state. Tectonophysics. 2011; 503(3-4): 195-200. doi: 10.1016/j. tecto.2011.02.012

12. Tong HM, Wang JJ, Zhao HT, et al. Mohr space and its application to the activation prediction of pre-existing weakness. Science China: Earth Sciences. 2014; 57(7): 1-10. doi: 10.1007/s11430-014-4860-1

13. Coelho S, Passchier C. Mohr-cyclides, a 3D representation of geological tensors: The examples of stress and flow. J Structural Geology. 2008; 30(5): 580-601. doi: 10.1016/j.jsg.2008.01.003

14. Brace WF. Mohr construction in the analysis of large geological strain. Geolological Society of America Bulletin. 1961; 72(7): 1059-79. doi: 10.1130/0016-7606

15. Ramsay JG. Folding and Fracturing of Rocks. McGraw-Hill, New York 1967.

16. Delaney PT, Pollard DD, Zioney Jl, MacKee EH. Field relations between dikes and joints: Emplacement processes and paleostress analysis. Journal of Geophysical Research. 1986; 91: 4920-38. doi: 10.1029/JB091iB05p04920 
17. Jolly RJH, Sanderson DJ. A Mohr circle construction for the opening of a pre-existing fracture. J Structural Geology. 1997; 19(6): 887-892. doi: 10.1016/S0191-8141(97)00014-X

18. Twiss RJ, Moores. Structural Geology. New York, W. H. Freeman and Company 1992.

19. Tong H, Cai D, Wu Y, Li X, Li X, Meng L. Activity criterion of pre-existing fabrics in non-homogeneous deformation domain. Science China (Earth Science). 2010; 53(8): 1115-25. doi: 10.1007/s11430-010-3080-6

20. Collettini C, Trippetta F. A slip tendency analysis to test mechanical and structural control on aftershock rupture planes. Earth and Planetary Science Letters. 2017; 255(3-4): 402-413. doi: 10.1016/j.epsl.2007.01.001

21. Bailey W, Ben-Zion Y. Statistics of Earthquake Stress Drops on a Heterogeneous Fault in an Elastic Half-Space. Bulletin of the Seismological Society of America. 2009; 99(3): 1786-1800.

22. Yin ZM, Ranalli G. Estimation of the frictional strength of faults from inversion of fault-slip data: a new method. J Structural Geology. 1995; 17(9): 1327-35. doi: 10.1016/0191-8141(95)00028-C

23. Wallace RE. Geometry of shearing stress and relation to faulting. J. Geol. 1951; 59: 118-130.

24. Bott MHP. The mechanics of oblique slip faulting. Geol.Mag. 1959; 96(2): 109-117.
25. McKenzie DP. The relation between fault plane solutions for earthquakes and the directions of the principal stresses. Bull.Seismol. Soc. Am. 1969; 59: 591-601.

26. Morley CK. A tectonic model for the Tertiary evolution of strike-slip faults and rift basins in SE Asia. Tectonophysics. 2002; 347(4): 189-215. doi: 10.1016/S0040-1951(02)00061-6

27. Haimson B, Rudnicki JW. The effect of the intermediate principal stress on fault formation and fault angle in siltstone. J Structural Geology. 2010; 32(11): 1701-11. doi: 10.1016/j.jsg.2009.08.017

28. Tong $H$, Koyi $H$, Huang $S$, Zhao $H$. The effect of multiple pre-existing weaknesses on formation and evolution of faults in extended sandbox models. Tectonophysics. 2014; 626: 197-212. doi: 10.1016/j. tecto.2014.04.046

29. Gudmundsson A, Simmenes TH, Larsen B, Philipp SL. Effects of internal structure and local stresses on fracture propagation, deflection, and arrest in fault zones. J. Struct. Geol. 2010; 32(11): 1643-55. doi: 10.1016/j. jsg.2009.08.013

30. Yin A. Mechanics of monoclonal systems in the Colorado plateau during the Laramide orogen. J. Geophys. Res. 1994; 99: 22043-58. 